\title{
GENERATION OF SH-TYPE WAVES IN LAYERED ANISOTROPIC ELASTIC MEDIA
}

\author{
P.C. PAL \\ Department of Mathematics \\ Indian School of Mines \\ Dhanbad - 826004, India \\ LOKENATH DEBNATH \\ Departments of Mathematics and Physics \\ East Carolina University \\ Greenville, N. C. 27832, U. S. A.
}

(Received November 8, 1978 and in revised form July 2, 1979)

ABSTRACT. A study is made of the generation of SH-type waves at the free surface of a layered anisotropic elastic medium due to an impulsive stress discontinuity moving with uniform velocity along the interface of the layered medium. The exact solution for the displacement function is obtained by the Laplace and Fourier transforms combined with the modified Cagniard method. The numerical results for an important special case at two different distances are shown graphically. The results of the present study are found to be in excellent agreement with those of isotropic elastic media.

KEY WOROS AND PHRASES. SH-type waves, anisotropic elastic waves, seismology and shearing stress discontinuity.

1980 Mathematics Subject Classification Code: 73015, $73020,73030$. 


\section{INTRODUCTION.}

In recent years an attention has been given to problems of generation and propagation of waves in anisotropic or inhomogeneous elastic solids. The solutions of these problems in various geometrical configurations, and in layered media are important in geophysics, seismology, acoustics and electromagnetism. Available information strongly suggests that layered media, crystals, and various new materials such as reinforced plastics, fibre-reinforced metals, and composite materials are essentially anisotropic and/or inhomogeneous in nature. Naturally, there is a growing need for elastodynamical analysis of anisotropic and/or inhomogeneous problems in elastic materials.

Some recent works on the generation and propagation of waves in layered isotropic or anisotropic elastic media may be relevant to mention with a brief description in order to indicate our motivation. Anderson [1] made an interesting study of elastic wave propagation in layered anisotropic media with applications. He discussed the period equations for waves of Rayleigh, Stoneley, and Love types. It was shown that anisotrophy can have a pronounced effect on both the range of existence and the shape of the dispersion curves. Finally, the single layer solutions in an anisotropic medium were generalized to n-layer media by the use of Haskell matrices.

Elastic properties are generally anisotropic (transversely isotropic) in sedimentary layers. Studies of Uhring and Van Melle [2], and Anderson and Harkrider [3] have indicated that anisotrophy is also present in the near surface layers, and in the crust and upper mantle regions of the earth. It has been observed that the transition region between the crust and mantle is not a single layer but is possibly formed by a set of thin layers. Nag [4] made an investigation of the generation of SH-type waves at the free surface of an isotropic elastic layered medium due to an impulsive stress discontinuity moving with constant velocity after creation along the interface of the medium. In a subsequent paper Nag and Pal [5] 
solved a problem similar to that of Nag with a shearing stress discontinuity at the interface of two layers of finite thickness overlying a semi-infinite medium of different elastic constants. Mention may also be made of the work on forced vibrations of an anisotropic elastic spherical shell due to an uniformly distributed Internal and external pressure by Sheehan and Debnath [6].

The purpose of this paper is to study the generation of SH-type waves at the free surface of a layered anisotropic elastic medium due to an impulsive stress discontinuity moving with uniform velocity along the interface of the layered medium. The displacement function is obtained for two different types of discontinuity in the shearing stress. The numerical solution for one case at two different distances is shown graphically. Some special cases of physical interest are examined.

\section{MATHEMATICAL FORMULATION OF THE PROBLEM.}

We consider an anisotropic elastic layer of thickness $h$ with elastic constants $L_{1}, N_{1}$ and $\rho_{1}$ over an anisotropic half-space with constants $L_{2}, N_{2}$ and $\rho_{2}$. The geometrical configuration of the anisotropic wave problem is depicted in Figure 1. With the z-axis directed vertically downwards, the transversely isotropic layered structure is referred to a rectangular Cartesian coordinate system with the origin at the interface $(z=0)$ of the layers I and II as shown in Figure 1 . The wave generating mechanisn is a shearing stress discontinuity which occurs suddenly at the interface and then moves with constant velocity $V$ in the positive $x$-direction along the interface. Since only the SH-type waves are considered, we can assume that the displacement fields $u=w=0$ and $v$ is a function of space variable $x, z$ and $t$.

Neglecting body forces and assuming small deformations, the equations of motion in the two layers can be written, with the usual notations, in the form

$$
\rho_{i} \frac{\partial^{2} v_{i}}{\partial t^{2}}=N_{i} \frac{\partial^{2} v_{i}}{\partial x^{2}}+L_{i} \frac{\partial^{2} v_{i}}{\partial z^{2}}, \quad t>0,
$$


where $1=1,2, v_{1}$ and $v_{2}$ represent the displacement functions in the layers I and II respectively.

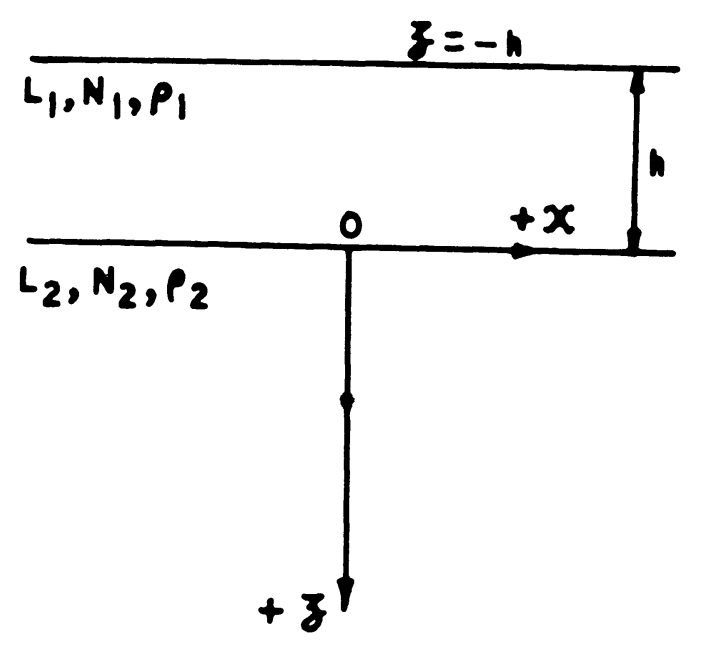

\section{GEOMETRY OF THE PROBLEM}

\section{FIG.1.}

The bourdary conditions are, in the usual notations,

$$
\begin{aligned}
& (\widetilde{y z})_{1}=0 \text { at } z:=-h, t>0 \\
& v_{1}=v_{2} \text { at } z=0, t>0 \\
& (\widetilde{y z})_{1}-(\widetilde{y z})_{2}=S(x, t) H(t) \text { at } z=0
\end{aligned}
$$

where $S(x, t)$ is a function of $x$ and $t ; H(t)$ is the Heaviside unit function of time $t$, and the shearing stress in an anisotropic medium is given by

$$
(\overbrace{\mathrm{yz}})_{i}=\mathrm{L}_{1} \frac{\partial \mathrm{v}_{1}}{\partial z}, 1=1,2
$$

We complete the formulation of the problem by assuming the appropriate initial conditions and the existence of the Laplace and Fourler transforms of the functions $v_{1}(x, z ; t)$ with respect to time $t$ and distance $x$ respectively. 


\section{THE SOLUTION OF THE PROBLW.}

The above problea can readily be solved by using the Laplace and Fourler transforms combined with the modified Cagniard method. The Laplace transform with respect to $t$ and the Pourier transform with respect to $x$ are defined by

$$
\frac{\tilde{v}}{v}(\xi, z ; p)=\int_{-\infty}^{\infty} e^{-1 \xi x} d x \int_{0}^{\infty} e^{-p t} v(x, z ; t) d t,
$$

where the tilda and the bar dewote the Laplace and the Fourfer transforms respectively. Application of the double transforns (3.1) to equations (2.1ab) give the solutions in the upper and lover layers with $v_{2} \rightarrow 0$ as $z+\infty$ in the form

$$
\begin{aligned}
& \bar{v}_{1}(x, z ; p)=\int_{-\infty}^{\infty}\left(A \cosh \eta_{s 1} z+B \sinh \eta_{s 1} z\right) e^{1 \xi x} d \xi, \\
& \bar{v}_{2}(x, z ; p)=\int_{-\infty}^{\infty} c \exp \left(1 \xi x-\eta_{s 2} z\right) d \xi,
\end{aligned}
$$

where the constants $A, B$ and $C$ are to be determined from the boundary conditions

$$
\begin{aligned}
(2.2) & -(2.4) ; \\
n_{s 1} & =\left(p^{2} / \beta_{11}^{2}+\xi^{2} \phi_{1}^{2}\right)^{\frac{1}{2}}, 1=1,2, \\
\beta_{11} & =\left(\frac{L_{1}}{\rho_{1}}\right)^{\frac{1}{2}}, \beta_{21}=\left(\frac{N_{1}}{\rho_{1}}\right)^{\frac{1}{2}},
\end{aligned}
$$

and $\phi_{1}=\beta_{21} / \beta_{11}$ is the an1sotropic parameter for the two layers $I$ and II.

It follows from the boundary conditions (2.2) - (2.3) that

$$
A=C, B \cosh \eta_{s 1} h=A \sinh \eta_{s 1} h \text {, }
$$

We now consider two different forms of the functions $s(x, t)$.

$$
\text { Case (1): } \left.\begin{array}{rl}
S(x, t) & =p, a \leq x \leq b+\nabla t \\
& =0, \text { elsewhere }
\end{array}\right\}
$$

where $P$ is a constant.

This case implies that the stress discontinulty is created in the region $x=a$ 
to $x=b$ and then expands with constant velocity $V$ in the $x$-direction. In particular, when $a=b=0$, the discontinuity is created at the origin and then expands with unfform velocity $\mathrm{V}$ in the $\mathrm{x}$-direction. When the disturbance expands in both directions after creation, this solution is to be added to the solution for negative values of $x$.

It follows from the transformed boundary condition (2.4) combined with (3.7) that

$$
\mathrm{BL}_{1} \eta_{s 1}+\mathrm{CL}_{2} \eta_{\mathrm{s} 2}=\frac{\mathrm{P}}{2 \pi \mathrm{p}}\left[\frac{\mathrm{e}^{-1 \xi \mathrm{a}}-\mathrm{e}^{-i \xi \mathrm{b}}}{i \xi}+\frac{\mathrm{e}^{-i \xi \mathrm{b}}}{1 \xi+\mathrm{p} / \mathrm{v}}\right] \text {, }
$$

With the aid of (3.6ab) and(3.8), we solve for the three constants A,B,C; and then write down the integral solution for the displacement function at the free surface $z=-h$ in the form

$$
\begin{aligned}
& \bar{v}_{1}(x,-h, p)=\frac{p}{2 \pi p} \int_{-\infty}^{\infty} \frac{\exp (1 \xi x)}{\left(L_{1} n_{s 1} \sinh \eta_{s 1} h+L_{2} n_{s 2} \cosh n_{s 1} h\right)} \times \\
& \times\left[\frac{e^{-i \xi x}-e^{-i \xi b}}{1 \xi}+\frac{e^{-1 \xi b}}{i \xi+p / V}\right] d \xi \\
& =\frac{P}{\pi p} \int_{-\infty}^{\infty} \frac{\exp (i \xi x-h \eta s 1)}{\left(L_{1} \eta_{s 1}+L_{2}{ }^{n} s 2\right.}\left[\frac{e^{-i \xi a}-e^{-i \xi b}}{i \xi}+\frac{e^{-i \xi b}}{i \xi+p / V}\right]\left(1-K e^{-2 h n} s 1\right)_{d \xi}^{-1} \text {, }
\end{aligned}
$$

where $\mathbf{K}(<1)$ represents the reflection parameter given by

$$
K=\frac{L_{1} n_{s 1}-L_{2}{ } 2}{L_{1}{ }^{n} 1^{+L} L^{n} s 2}
$$

Using the inverse Laplace transform, we can rewrite (3.9) in a convenient form

$$
v_{1}(x,-h ; p)=L^{-1}\left(I_{1}+I_{2}+I_{3}\right)
$$

where $L^{-1}$ stands for the inverse Laplace transform and

$$
I_{1}=\frac{P}{\pi p} \int_{-\infty}^{\infty} \frac{\left(1-K e^{-2 h n} s 1\right)^{-1}}{i \xi\left(L_{1} n_{s 1}+L_{2}{ }^{n} s\right)} \exp \left(1 \xi x_{1}-h \eta_{s 1}\right) d \xi,
$$




$$
\begin{aligned}
& I_{2}=\frac{P}{\pi p} \int_{-\infty}^{\infty} \frac{\left(1-K e^{-2 h n} s 1\right)^{-1}}{i \xi\left(L_{1}{ }^{n} 1^{+L} 2^{n} s 2\right)} \exp \left(i \xi x_{2}-h n_{s 1}\right) d \xi \text {, }
\end{aligned}
$$

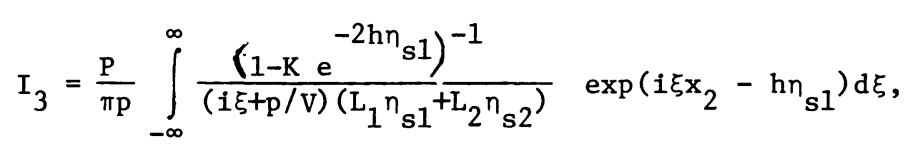

with $\mathrm{x}_{1}=\mathrm{x}-\mathrm{a}$ and $\mathrm{x}_{2}=\mathrm{x}-\mathrm{b}$.

In order to evaluate the Laplace inversion integral, we shall use the modified Cagniard method due to Garvin [7] who discussed the contour integration and mapping in detail. It may be fair to avoid duplication of mathematical analysis similar to that of Garvin, and to quote some necessary results from that paper without proof. We next substitute $\xi=\mathrm{p} \zeta / \beta_{11}$ in $(3.4 \mathrm{ab})$ and 3.10 ) so that

$$
\begin{aligned}
& \text { and } \eta_{s 1}=\frac{p}{\beta_{11}}\left(1+\zeta^{2} \phi_{1}^{2}\right)^{\frac{1}{2}}, n_{s 2}=\frac{p}{\beta_{11}}\left(\frac{\beta_{11}}{\beta_{12}{ }^{2}}+\zeta^{2} \phi_{2}{ }^{2}\right)^{\frac{1}{2}} \\
& \quad \mathrm{~L}=\frac{\mathrm{L}_{1}\left(1+\zeta^{2} \phi_{1}{ }^{2}\right)^{\frac{1}{2}}-\mathrm{L}_{2}\left(\frac{\beta_{11}}{\beta_{12}}+\zeta^{2} \phi_{2}{ }^{2}\right)^{\frac{1}{2}}}{\mathrm{~L}_{1}\left(1+\zeta^{2} \phi_{1}{ }^{2}\right)^{\frac{1}{2}}+\mathrm{L}_{2}\left(\frac{\beta_{11}}{\beta_{12}{ }^{2}}+\zeta^{2} \phi_{2}{ }^{2}\right)^{\frac{1}{2}}},
\end{aligned}
$$

We thus obtain

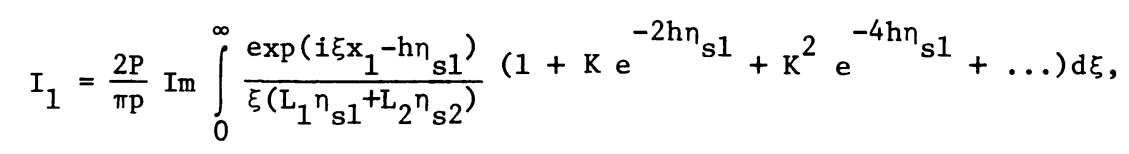

where $\eta_{s 1}$ and $\eta_{s 2}$ are given by $(3.15 \mathrm{ab})$.

Denoting the first term of $I$, in (3.17) by $I_{11}$, we get

$$
I_{11}=\frac{2 P}{\pi p^{2} \rho_{1} \beta_{11}} \operatorname{Im} \int_{0}^{\infty} \frac{\exp \left[-\mathrm{p}\left\{-i \zeta \mathrm{x}_{1}+\mathrm{h}\left(1+\zeta^{2} \phi_{1}{ }^{2}\right)^{\frac{1}{2}} \div \beta_{11}\right] \mathrm{d} \zeta\right.}{\zeta\left[\left(1+\zeta^{2} \phi_{1}{ }^{2}\right)^{\frac{1}{2}}+\frac{\mathrm{L}_{2}}{\mathrm{~L}_{1}}\left(\frac{\mathrm{B}_{11}{ }^{2}}{\mathrm{~B}_{12}{ }^{2}}+\zeta^{2} \phi_{2}{ }^{2}\right)^{\frac{1}{2}}\right]}
$$

The integrand of (3.18) has singularities at $\zeta=0, \pm 1 / \phi_{1}$ and \pm i $\beta_{11} / \phi_{2} \beta_{12}$. Setting 


$$
t=\left[-i \zeta x_{1}+h\left(1+\zeta^{2} \phi_{1}^{2}\right)^{\frac{1}{2}}\right] \beta_{11}^{-1}
$$

so that the inversion gives

$$
\zeta(t)=\frac{\beta_{11}}{\left(x_{1}{ }^{2}+h^{2} \phi_{1}^{2}\right)}\left[1 t x_{1}+h\left\{t^{2}-\left(x_{1}^{2}+h^{2} \phi_{1}^{2}\right) \beta_{11}^{-2}\right\}^{\frac{1}{2}}\right],
$$

The singularities in the $\zeta$-plane are shown in Figure 2, and the mapping and contour in the t-plane are depicted in Figure 3. Making reference to these F1gures and to the paper [4], we find

$$
L^{-1} I_{11}=\frac{2 P}{\pi \rho_{1} \beta 11} \int_{0}^{t}(t-\tau) G_{1,1}\left[\zeta_{1,1}(\tau)\right] d \tau,
$$

where $L[t H(t)]=\frac{1}{p^{2}}$ and

$$
\begin{aligned}
G_{1, t}[\zeta(t)] & =\operatorname{Im}\left[\left\{1+\phi_{1}{ }^{2} \zeta_{1,1}{ }^{2}(t)\right\}^{\frac{1}{2}}+\frac{L_{2}}{L_{1}}\left\{\frac{\beta_{11}{ }^{2}}{B_{12}{ }^{2}}+\phi_{2}{ }^{2} \zeta_{1,1}{ }^{2}(t)\right\}^{\frac{1}{2}}\right] \\
& \times \zeta_{1,1}{ }^{-1} \cdot \frac{d}{d t} \zeta_{1,1}(t) \cdot H\left\{t-\left(x_{1}{ }^{2}+h^{2} \phi_{1}{ }^{2}\right)^{\frac{1}{2}} \beta_{11}{ }^{-1}\right\},
\end{aligned}
$$

Since $h \phi_{1} \beta_{11}^{-1}<t<\left(x_{1}{ }^{2}+h^{2} \phi_{1}{ }^{2}\right)^{\frac{1}{2}} \beta_{11}{ }^{-1}$, the expression

$$
\left[\left\{1+\zeta_{1,1}{ }^{2}(t) \phi_{1}{ }^{2}\right\}^{\frac{1}{2}}+\frac{L_{2}}{L_{1}}\left\{\frac{\beta_{11}{ }^{2}}{\beta_{12}{ }^{2}}+\phi_{2}{ }^{2} \zeta_{1,1}{ }^{2}(t)\right\}^{\frac{1}{2}}\right] \text { is real. In general, it turns }
$$

out that

$$
L^{-1} I_{1, n}=\frac{2 P}{\pi \rho_{1} \beta_{11}} \int_{0}^{t}(t-\tau) G_{1, n}\left[\zeta_{1, n}(\tau)\right] d \tau,
$$

where

$$
\begin{aligned}
G_{1, n}\left[\zeta_{1 . n}(t)\right] & =\operatorname{Im}\left[\left\{1+\phi_{1}{ }^{2} \zeta_{1, n}{ }^{2}(t)\right\}^{\frac{1}{2}}+\frac{L_{2}}{L_{1}}\left\{\frac{\beta_{11}}{\beta_{12}{ }^{2}}+\phi_{2}{ }^{2} \zeta_{1, n}{ }^{2}(t)\right\}^{\frac{1}{2}}\right] \\
& \times \zeta_{1, n}{ }^{-1} \frac{d \zeta_{1, n}{ }^{(t)}}{d t} H\left\{t-\left(x_{1}{ }^{2}+n^{2} h^{2} \phi_{1}{ }^{2}\right)^{\frac{1}{2}} \beta_{11}{ }^{-1}\right\},
\end{aligned}
$$

and

$$
\zeta_{1, n}(t)=\frac{\beta_{11}}{\left(x_{1}{ }^{2}+n^{2} h^{2} \phi_{1}{ }^{2}\right)}\left[i x, t+n h\left\{t^{2}-\left(x_{1}{ }^{2}+n^{2} h^{2} \phi_{1}{ }^{2}\right) \beta_{11}{ }^{-2}\right\}^{\frac{1}{2}}\right], n=1,3,5 \ldots
$$




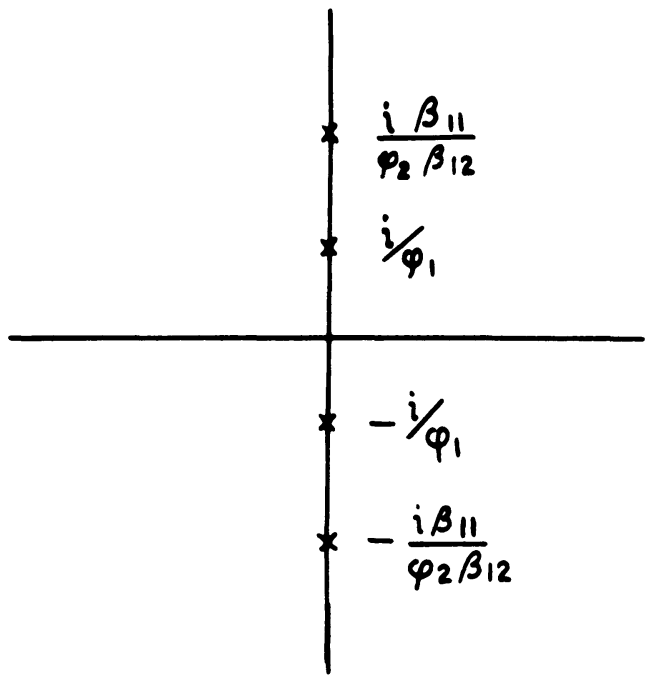

$\zeta$ - plane showing the singularities

FIG. 2 .

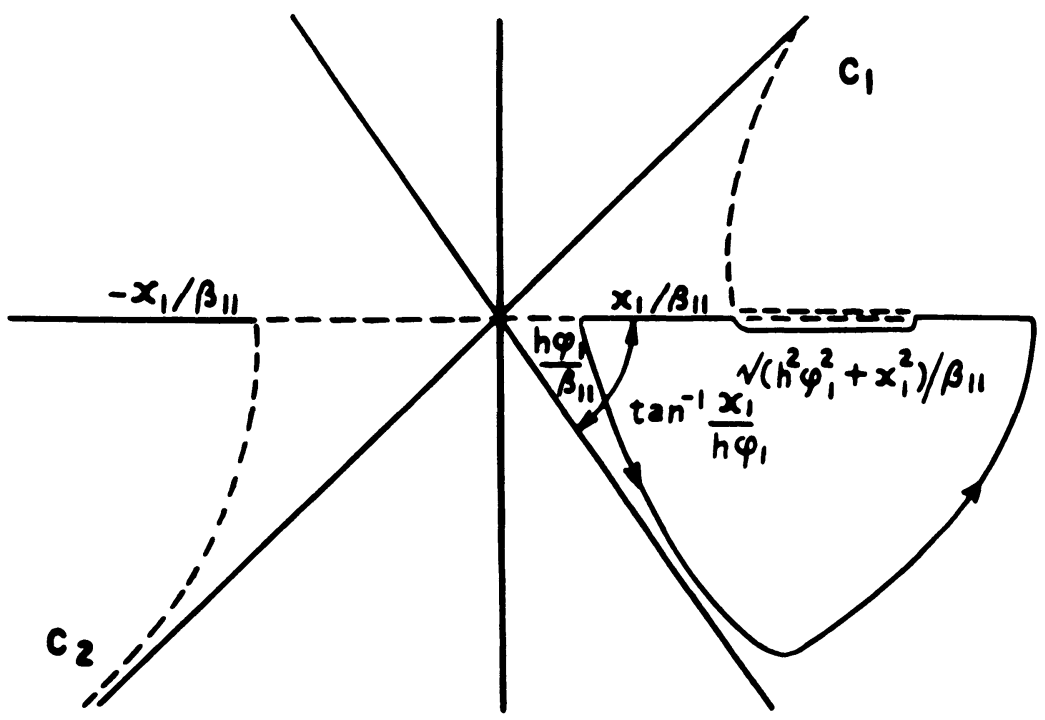

t - PLANE ShOWING THE MAPPING AND THE CONTOUR FIG. 3 . 
so that

$$
L^{-1} I_{1}=\sum_{n=1,3,5 \ldots} L^{-1} I_{1, n}
$$

Sinilarly, we obtain

$$
L^{-1} I_{2}=\sum_{n=1,3,5} L^{-1} I_{2, n}
$$

A similar procedure gives that

$$
L^{-1} I_{3}=\sum_{n=1,3,5 \ldots} L^{-1} I_{3, n}
$$

where

$$
\begin{aligned}
& L^{-1} I_{3, n}=\frac{2 P}{\pi \rho_{1} \beta_{11}} \int_{0}^{t}(t-\tau) G_{3, n}\left[\zeta_{2, n}(\tau)\right] d \tau, \\
& G_{3, n}\left[\zeta_{2, n}(t)\right]=\operatorname{Re}\left[\left\{1+\phi_{1}{ }^{2} \zeta_{2, n}{ }^{2}(t)\right\}^{\frac{1}{2}}+\frac{L_{2}}{L_{1}}\left\{\frac{\beta_{11}}{\beta_{12}{ }^{2}}+\phi_{2}{ }^{2} \zeta_{2, n}{ }^{2}(t)\right\}^{\frac{1}{2}}\right] \\
& \times\left[\frac{\beta_{11}}{V}+i \zeta_{2, n}(t)\right]^{-1} \mathrm{~K}^{\frac{n-1}{2}} \zeta_{2, n} \frac{d \zeta_{2, n}(t)}{d t} H\left\{t-\left(x_{2}{ }^{2}+n^{2} h^{2} \phi_{1}{ }^{\frac{1}{2}}\right)^{\beta_{11}}\right\}^{-1},
\end{aligned}
$$

and $\zeta_{2, n}(t)$ is given by (3.25) with $x_{2}$ in place of $x_{1}$.

Finally, a simple combination of the results (3.26) - (3.28) gives the exact value of the free surface displacement field $v_{1}(x,-h, t)$.

Case (ii) : $\quad S(x, t)=P h \delta(x-V t)$,

where $\mathrm{P}$ is a constant, $\delta(\mathrm{r})$ is the Dirac distribution and a factor $\mathrm{h}$ is included in (3.31) so that $P$ has the dimension of stress.

The boundary condition (2.4) given

$$
\mathrm{BL}_{1} n_{\mathrm{s} 1}+\mathrm{CL}_{2} n_{\mathrm{s} 2}=\frac{\mathrm{Ph}}{2 \pi \mathrm{V}(1 \xi+\mathrm{P} / \mathrm{V})} \text {, }
$$

Solving A, B, and C from (3.6 ab) and (3.32), we obtain the solution as

$$
\bar{v}_{1}(x,-h ; p)=\frac{P h}{\pi V} \int_{-\infty}^{\infty} \frac{\left(1+K e^{-2 \eta} s 1^{h}+\ldots . . . .\right)}{\left(L_{1} \eta_{s 1}+L_{2} \eta_{s 2}\right)(i \xi+p / v)} \exp \left(i \xi x-h n_{s 1}\right) d \xi
$$


A procedure similar to that of Case (i) gives the solution in the form

$$
v_{1}(x,-h, t)=\frac{2 P h}{\pi V \beta_{11}} \sum_{n=1,3,5 \ldots .} \int_{0}^{t} G_{n}\left[\zeta_{n}(\tau)\right] d \tau,
$$

where

$$
\begin{aligned}
& G_{n}\left[\zeta_{n}(t)\right]=\operatorname{Re}\left[\left\{1+\phi_{1}{ }^{2} \zeta_{n}{ }^{2}(t)\right\}^{\frac{1}{2}}+\frac{L_{2}}{L_{1}}\left\{\frac{\beta_{11}{ }^{2}}{\beta_{12}{ }^{2}}+\phi_{2}{ }^{2} \zeta_{n}{ }^{2}\right\}^{\frac{1}{2}}\right] \times \\
& \times\left[\frac{\beta_{11}}{V}+i \zeta_{n}(t)\right]^{-1} \frac{d \zeta_{n}(t)}{d t} k^{\frac{n-1}{2}} \zeta_{n}(t) H\left\{t-\left(x^{2}+n^{2}{ }^{2} \phi_{1}{ }^{2}\right)^{\frac{1}{2}} B_{11}{ }^{-1}\right],
\end{aligned}
$$

and

$$
\zeta_{n}(t)=\frac{\beta_{11}}{\left(x^{2}+n^{2} h^{2} \phi_{1}{ }^{2}\right)}\left[1 t x+n h\left\{t^{2}-\left(x^{2}+n^{2} h^{2} \phi_{1}{ }^{2}\right) \beta_{11}{ }^{-2}\right\}^{\frac{1}{2}}\right],
$$

If the stress discontinuity is taken as $H(x)-H(x-V t)$ in place of $\delta(x-V t)$, the coresponding result on the right hand side of (3.33) differ only by a constant factor from $I_{3}$ (with $a=b=0$ ) in (3.12) - (3.14). 4. NUMERICAL RESULTS :

It is of interest to consider the inftial behavior of the displacement field $v_{1}(x,-h, t)$ for case (ii) numerically.

Following $\mathrm{Nag}$ and $\mathrm{Pal}$ [5], motion due to each of the pulses at the instant of their arrival has been investigated. We consider the following numerical values: $\phi_{1}=0.9, \phi_{2}=1.1, \mathrm{~L}_{2} / \mathrm{L}_{1}=1.33, \mathrm{\beta}_{11} / \mathrm{V}=1.21, \mathrm{\beta}_{11} / \mathrm{B}_{12}=1.02$.

The initial behavior of $v_{1}(x,-h, t)$ at two different distances is examined.

(1) When $x=5 h, t=\frac{\tau h}{\beta_{11}}$, for six initial values, we have

$$
\begin{aligned}
& K_{1} v_{1}(x,-h, t) \approx\left[A^{0}\left(\theta_{1}\right) \cosh ^{-1} \frac{\tau}{5.2} H(\tau-5.2)+\right. \\
+ & A^{0}\left(\theta_{3}\right) \cosh ^{-1} \frac{\tau}{6.3} H(\tau-6.3)+A^{0}\left(\theta_{5}\right) \cosh ^{-1} \frac{\tau}{8.2} H(\tau-8.2) \\
+ & A^{0}\left(\theta_{7}\right) \cosh ^{-1} \frac{\tau}{10.1} H(\tau-10.1)+A^{0}\left(\theta_{9}\right) \cosh ^{-1} \frac{\tau}{12.6} H(\tau-12.6)+ \\
+ & \left.A^{0}\left(\theta_{11}\right) \cosh ^{-1} \frac{\tau}{15.2} H(\tau-15.2)\right],
\end{aligned}
$$


where $K_{1}=\frac{2.42 \mathrm{P} \rho_{1}}{\pi \cdot \beta_{11}}$,

and

$$
\begin{aligned}
\mathrm{A}^{\circ}\left(\theta_{\mathrm{n}}\right)= & \operatorname{Re} \frac{\left[\left(1-1.76 \cos ^{2} \theta_{n}\right)^{1 / 2}-0.95\left(1.04-2.1 \cos ^{2} \theta_{n}\right)^{1 / 2}\right]^{\frac{\mathrm{n}-1}{2}} \sin \theta_{n}}{\left[\left(1-1.76 \cos ^{2} \theta_{n}\right)^{1 / 2}+0.95\left(1.04-2.1 \cos ^{2} \theta_{n}\right)^{1 / 2}\right]^{\frac{n-3}{2}}\left(1.2-\cos \theta_{n}\right)}, \\
n & =1,3,5, \ldots \ldots
\end{aligned}
$$

(2) When $x=10 \mathrm{~h}, t=\frac{\tau h}{\beta_{11}}$, for six initial values, we have

$$
\begin{aligned}
& \mathrm{K}_{1} \mathrm{v}_{1}(\mathrm{x},-\mathrm{h}, \mathrm{t}) \approx\left[\mathrm{A}^{\mathrm{O}}\left(\theta_{1}\right) \cosh ^{-1} \frac{\tau}{10.08} \mathrm{H}(\tau-10.08)+\right. \\
& +\mathrm{A}^{\mathrm{O}}\left(\theta_{3}\right) \cosh ^{-1} \frac{\tau}{10.71} \mathrm{H}(\tau-10.71)+ \\
& +\mathrm{A}^{\mathrm{O}}\left(\theta_{5}\right) \cosh ^{-1} \frac{\tau}{11.94} \mathrm{H}(\tau-11.94)+\mathrm{A}^{\mathrm{O}}\left(\theta_{7}\right) \cosh ^{-1} \frac{\tau}{13.64} \mathrm{H}(\tau-13.64)+ \\
& \left.+A^{0}\left(\theta_{9}\right) \cosh ^{-1} \frac{\tau}{15.5} H(\tau-15.5)+A^{0}\left(\theta_{11}\right) \cosh ^{-1} \frac{\tau}{17.62} H(\tau-17.62)\right],
\end{aligned}
$$

where $A^{0}\left(\theta_{n}\right), n=1,3,5, \ldots \ldots$ are given by $(4.3)$.

Values of $A^{0}\left(\theta_{n}\right), n=1,3,5, \ldots$ for $x=5 h$ and $x=10 h$ are tabulated below:

\section{TABLE 1 .}

\begin{tabular}{lllllll}
\hline $\mathrm{A}^{0}\left(\theta_{\mathrm{n}}\right)$ & $\mathrm{A}^{0}\left(\theta_{1}\right)$ & $\mathrm{A}^{0}\left(\theta_{3}\right)$ & $\mathrm{A}^{0}\left(\theta_{5}\right)$ & $\mathrm{A}^{0}\left(\theta_{7}\right)$ & $\mathrm{A}^{0}\left(\theta_{9}\right)$ & $\mathrm{A}^{0}\left(\theta_{11}\right)$ \\
\hline $\mathrm{x}=5 \mathrm{~h}$ & 0.74 & -0.301 & 0.052 & -0.122 & 0.0009 & -0.00012 \\
\hline $\mathrm{x}=10 \mathrm{~h}$ & 0.23 & -0.02 & 0.0165 & -0.0035 & 0.00055 & -0.0002 \\
\hline
\end{tabular}

\section{DISCUSSION AND CONCLUSIONS.}

The exact form of the displacement field due to physically realistic shearing stress discontinuity has been obtained. The value of $k_{1} v_{1}(x,-h, t)$ for $x=5 h$ and $x=10 \mathrm{~h}$ have been plotted against $\tau_{1}=\tau-\tau_{0}$ where $\tau_{0}$ is the time at which the disturbances arrive at the point of observation. The values of for 


$$
\begin{array}{r}
{\left[\tau_{1} \sim k_{1} v_{1}\right] G \text { rash }} \\
\tau_{1}=\tau-\tau_{0} ; \tau=\frac{t \beta_{1}}{h}
\end{array}
$$

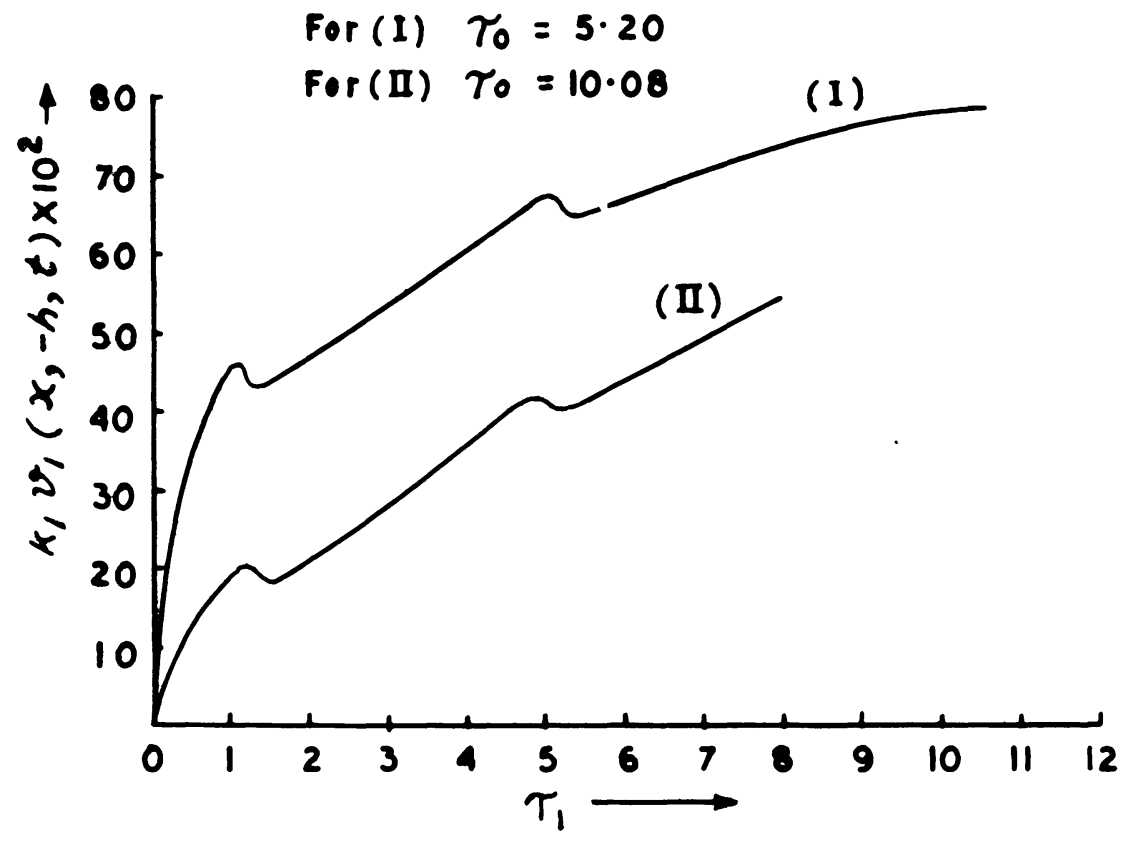

FIG.A.

$x=5 h$ and $x=10 h$ are 5.2 and 10.08 respectively. These results are shown in Figure 4. It is clear from Figure 4 that the curves start from the origin and undergo sharp changes in their slopes with sudden jerking as the different pulses arrive after undergoing reflections from the boundaries of the anisotropic layers. These changes occur for $x=5 h$ at $\tau=\left(5^{2}+0.81 n^{2}\right)^{1 / 2}, n=1,5,9, \ldots ;$ and for $x=10 \mathrm{~h}$ at $\tau=\left(10^{2}+0.81 \mathrm{n}^{2}\right)^{1 / 2}, \mathrm{n}=1,5,9, \ldots$. We a1so infer that the contribution due to the third pulse for $x=10 \mathrm{~h}$ will arrive in a shorter time than for $x=5 h$.

In addition to above two special disturbances, the present analysis incorporates other forms of physically realistic stress discontinuity for the generation of $\mathrm{SH}$ 
type waves in both isotropic and anisotropic elastic media.

In the case of the layered isotropic media, $\phi_{1}=1,1=1,2$; the results of the present analysis reduce to those of Nag [4] for the corresponding isotropic problem.

Finally the shearing stress discontinuity is always associated with the propogation of cracks in earthquakes. Hence the present study has direct applications to geophysics and seismology. In addition, the present study is of interest in connection with the propagation of cracks in sedimentary layers, and in particular in elastic layers having anisotropic property in general.

ACKNOWLEDGEMENT. The authors express their grateful thanks to Professor M. Bath, University of Uppsala, Sweden and to Professor K. R. Nag for their help and encouragement. This work was partially supported by the East Carolina University Research Committee.

\section{REFERENCES}

1. Anderson, D. L., Wave Propagation in Anisotropic Media, J. Goephys. Res. $\underline{66}$ (1961) 2953-2963.

2. Uhring, L. F. and Van Melle, E. M., Velocity Anisotrophy in the Stratified Media, Geophysics 21 (1955) 774-779.

3. Anderson, D. L. and Harkrider, D. G., The effect of anisotropy on Continental and Oceanic Surface wave dispersion (abstract) J. Geophys. Res. 67 (1962) 1627 .

4. Nag, K. R., Generation of SH-type wave due to stress Discontimuity in a layered elastic media, Quart. J. Mech. and App1. Math. 16 (1963) 295-303.

5. Nag, K. R. and Pal, P. C., The Disturbance of SH-type due to shearing stress Discontinuity at the Interface of two layers overlying a semi-infinite medium, Arch. Mech. 29 (1977) 821-827.

6. Sheehan, J. and Debnath, L., Forced Vibrations of an Anisotropic Elastic Sphere, Arch. Mech 24 (1972) 117-125.

7. Garvin, W. W., Exact Transient Solution of the Buried Line Source Problem, Proc. Roy. Soc. Lond. A234 (1956) 528-541. 


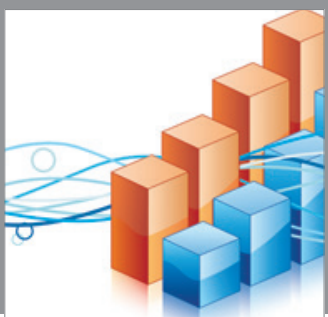

Advances in

Operations Research

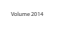

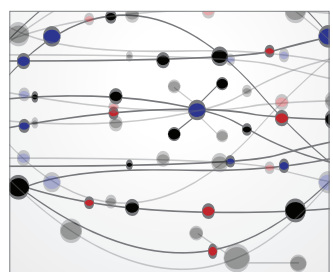

\section{The Scientific} World Journal
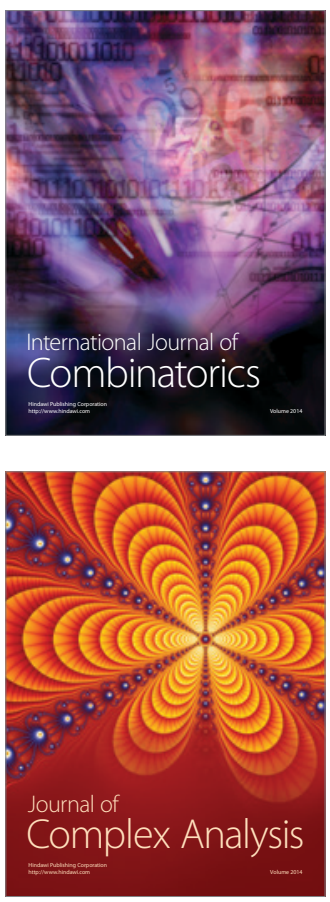

International Journal of

Mathematics and

Mathematical

Sciences
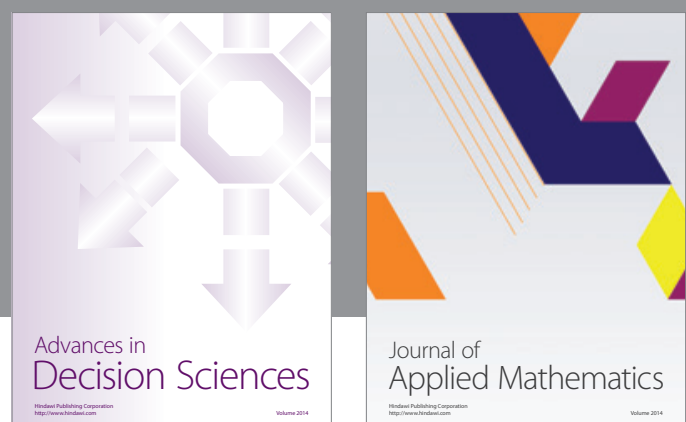

Journal of

Applied Mathematics
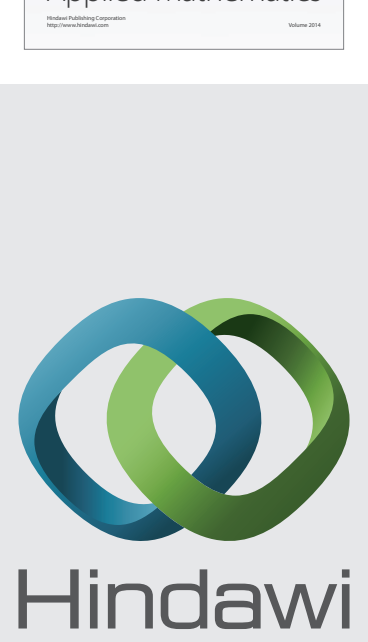

Submit your manuscripts at http://www.hindawi.com
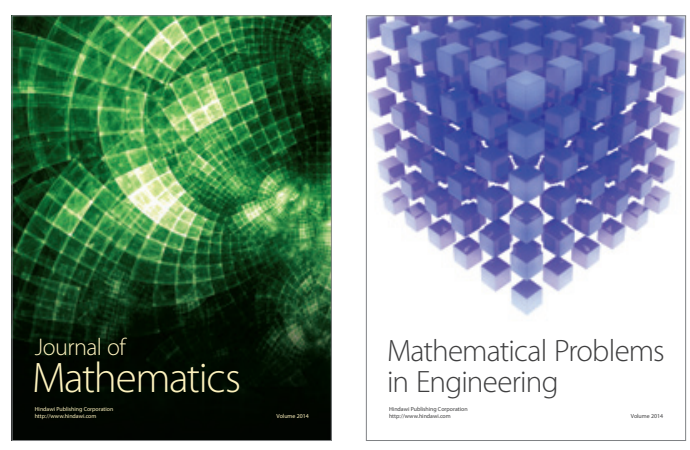

Mathematical Problems in Engineering
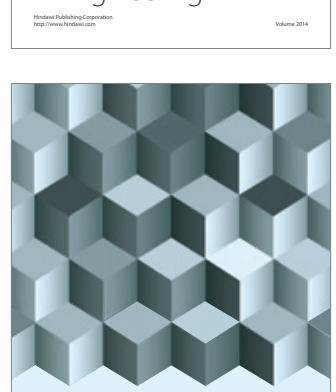

Journal of

Function Spaces
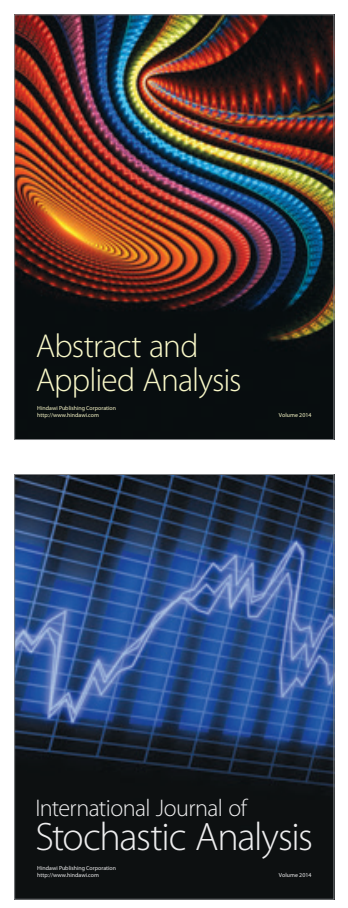

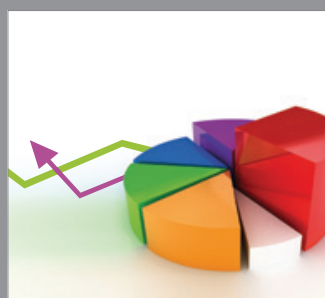

ournal of

Probability and Statistics

Promensencen
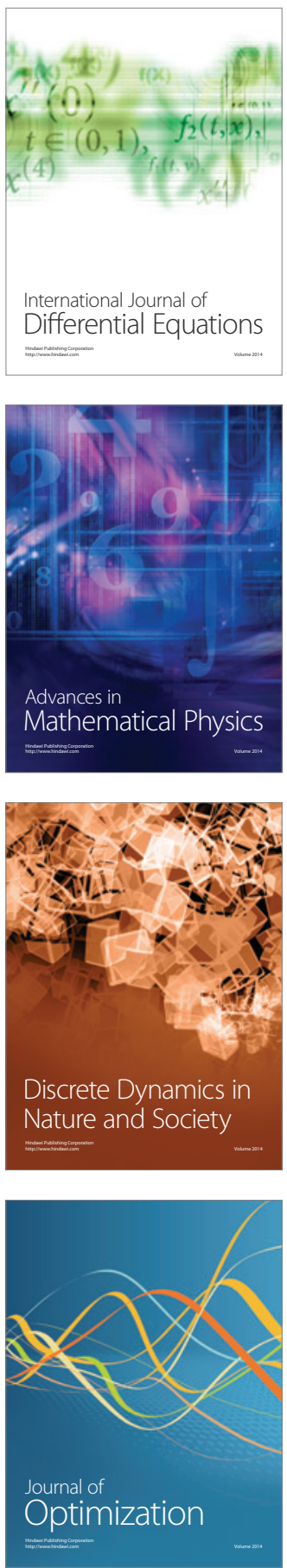